\title{
Impacto de la pandemia por COVID-19 en la práctica dermatológica en Colombia. Encuesta nacional
}

\author{
Claudia Marcela Arenas-Soto'; Laura Carolina Camacho-Pérez²; Camilo Andrés \\ Morales-Cardona3; Gloria Sanclemente-Mesa ${ }^{4}$
}

\section{RESUMEN}

Introducción: la pandemia por SARS-CoV-2 ha causado una interrupción en la prestación de los servicios de salud de todo el mundo, con lo que ha modificado el ejercicio de la medicina. La dermatología, una especialidad medicoquirúrgica, en la que existe contacto estrecho con el paciente, ha experimentado reducción en el número de consultas y cancelación de procedimientos quirúrgicos y dermocosméticos, en cumplimiento de las recomendaciones y lineamientos nacionales e internacionales.

Materiales y métodos: estudio transversal descriptivo por medio de una encuesta aplicada por vía electrónica a los dermatólogos colombianos, miembros de la Asociación Colombiana de Dermatología y Cirugía Dermatológica. El objetivo de este estudio fue determinar la magnitud del impacto de la pandemia por SARS-CoV-2 en la práctica de los dermatólogos colombianos.

Resultados: el 74\% de los encuestados reportó suspensión de la consulta dermatológica, el 83\% de procedimientos dermatológicos y solo un 6\% atendía pacientes de urgencias durante el confinamiento obligatorio. Secundario a la pandemia por COVID-19, un 79\% de los dermatólogos colombianos introdujo la modalidad de teleconsulta y los costos asociados al cumplimiento de las normas de bioseguridad generaron gastos imprevistos para el 20\% de los encuestados.

Conclusiones: la situación actual es un gran reto para la especialidad, que no solo debe mitigar el riesgo biológico derivado del retorno a sus actividades presenciales, sino conocer e implementar medidas preventivas que permitan proteger la salud y el bienestar común, mientras se fortalecen las condiciones para un ejercicio seguro y de calidad, apoyado en las nuevas tecnologías.

PALABRAS CLAVE: COVID-19; Dermatología; Pandemia; Telemedicina.

1. Dermatóloga. Especialista en Docencia Universitaria, Universidad Militar Nueva Granada. ORCID https://orcid.org/oooo-0003-3689-5355

2. Residente de dermatología, Universidad Nacional de Colombia. ORCID https://orcid.org/oooo-0003-3506-7339

3. Dermatólogo, Hospital Universitario, Centro Dermatológico Federico Lleras Acosta E.S.E. Pontificia Universidad Javeriana. ORCID https://orcid.org/oooo-0oo2-3187-3272

4. MD, MSc, PhD. Grupo de Investigacion Dermatológica (GRID), Instituto de Investigaciones Médicas, Universidad de Antioquia, Medellín, Colombia. ORCID https://orcid.org/oooo-0002-1555-2751

Correspondencia: Claudia Marcela Arenas-Soto; email: draclaudiaarenas@gmail.com

Recibido: 20/10/20; aceptado: $20 / 10 / 20$

Cómo citar: Arenas, CM; Camacho, LC; Morales, CA; Sanclemente, G. Impacto de la pandemia por COVID-19 en la práctica dermatológica en Colombia. Encuesta nacional. Rev Asoc Colomb Dermatol. Vol 28(4): octubre - diciembre, 2020, 318-324.

DOI: https://doi.org/10.29176/2590843X.1551

Financiación: ninguna, conflictos de interés: ninguno 


\section{THE IMPACT OF THE COVID-19 PANDEMIC ON DERMATOLOGICAL PRACTICE IN COLOMBIA: A NATIONAL SURVEY}

\section{SUMMARY}

Introduction: The SARS-CoV-2 pandemic has caused an interruption in the provision of health services around the world, thus modifying the practice of medicine. Dermatology, a medical-surgical specialty in which there is close contact with the patient, has experienced a reduction in the number of consultations, cancellation of surgical and dermo-cosmetic procedures, in compliance with national and international recommendations and guidelines.

Materials and methods: Descriptive cross-sectional study by means of a survey applied electronically to Colombian dermatologists, members of the Colombian Association of Dermatology and Dermatological Surgery. The objective of this study was to determine the magnitude of the impact of the SARS-CoV2 pandemic on the practice of Colombian dermatologists.

Results: $74 \%$ of the respondents had suspension of the dermatological consultation, $83 \%$ of dermatological procedures and only $6 \%$ assisted emergency patients during the mandatory confinement. Secondary to the COVID-19 pandemic, 79\% of Colombian dermatologists introduced the telemedicine modality and the costs associated with compliance with biosafety regulations generated unforeseen expenses for $20 \%$ of those surveyed.

Conclusions: The current situation is a great challenge for Dermatology, which must not only mitigate the biological risk derived from the return to its face-to-face activities, but also know and implement preventive measures that protect health and common welfare, while strengthening the conditions for a safe and quality exercise, supported by new technologies.

KEY WoRDS: COVID-19; Dermatology; Pandemic; Telemedicine.

\section{INTRODUCCIÓN}

La pandemia por SARS-CoV-2 ha modificado el ejercicio de la medicina, en particular aquellas especialidades con competencias quirúrgicas, debido al incremento en el riesgo de contagio por exposición a las secreciones del paciente ${ }^{(1)}$. La dermatología no ha sido ajena a la pandemia y muchos especialistas han tenido que adoptar nuevas condiciones para su ejercicio profesional, utilizando herramientas tecnológicas como la telemedicina, además de invertir tiempo y recursos para capacitarse en el uso correcto de equipo de protección personal (EPP) y prácticas seguras, con el objetivo de prevenir, contener y mitigar el riesgo de contagio que representa la atención presencial de sus pacientes ${ }^{(2-5)}$. Asimismo, la reducción en el número de consultas y la cancelación de procedimientos electivos, considerados no indispensables, ha generado un impacto negativo en los ingresos de los dermatólogos de todo el mundo ${ }^{(6)}$.
La disminución en la demanda de la especialidad se ha documentado en varios países, tanto en los servicios de consulta externa como en los de atención hospitalaria. En Italia, las consultas dermatológicas disminuyeron entre $80 \%$ y $90 \%$ durante el pico de la pandemia, probablemente debido a las medidas de aislamiento y restricción en la movilidad para frenar la curva de contagio $^{(1)}$.

Teniendo en cuenta lo anterior, se realizó una encuesta con el objetivo de conocer y analizar el impacto de la pandemia en el ejercicio profesional de un grupo de dermatólogos colombianos.

\section{MATERIALES Y MÉTODOS}

Estudio transversal descriptivo por medio de una encuesta realizada entre el 17 y el 29 de abril de 2020, período en el que toda la población colombiana se 
encontraba en aislamiento preventivo obligatorio. La encuesta fue aplicada por vía electrónica a los dermatólogos colombianos, miembros de la Asociación Colombiana de Dermatología y Cirugía Dermatológica. Se obtuvieron datos socioeconómicos, laborales y geográficos de cada encuestado. El análisis se realizó en el programa estadístico IBM $®$ SPSS $®$ Statistics. Para las variables cualitativas se hizo un análisis descriptivo con frecuencias y moda, y para las variables cuantita- tivas se analizaron medidas de tendencia central con promedios y valores máximo y mínimo.

\section{RESULTADOS}

Los dermatólogos colombianos diligenciaron 371 encuestas. El 36\% tenía entre 31 y 39 años y el 29\% entre 40 y 48 años; el $74 \%$ fueron mujeres. La mayoría de los dermatólogos ejercen en la ciudad de Bogotá (tabla 1).

Tabla 1. Distribución geográfica laboral de los dermatólogos

\begin{tabular}{|c|c|c|}
\hline Ciudad & Frecuencia & Porcentaje \% \\
\hline Armenia & 7 & 1,9 \\
\hline Barranquilla & 13 & 3,5 \\
\hline Bogotá & 131 & 35,3 \\
\hline Bucaramanga & 15 & 4 \\
\hline Cali & 30 & 8,1 \\
\hline Cartagena & 3 & 0,8 \\
\hline Cúcuta & 1 & 0,3 \\
\hline lbagué & 8 & 2,2 \\
\hline Leticia & 1 & 0,3 \\
\hline Manizales & 8 & 2,2 \\
\hline Medellín & 81 & 21,8 \\
\hline Montería & 3 & 0,8 \\
\hline Neiva & 3 & 0,8 \\
\hline Pasto & 1 & 0,3 \\
\hline Pereira & 14 & 3,7 \\
\hline Santa Marta & 4 & 1,1 \\
\hline Valledupar & 1 & 0,3 \\
\hline Villavicencio & 7 & 1,9 \\
\hline Espinal (Tolima) & 1 & 0,3 \\
\hline Regiones de Antioquia & 3 & 0,8 \\
\hline Regiones de Boyacá & 4 & 1,1 \\
\hline Regiones de Cundinamarca & 8 & 2,2 \\
\hline Regiones de Santander & 4 & 1,1 \\
\hline Regiones del Valle del Cauca & 7 & 1,9 \\
\hline Quibdó & 3 & 0,8 \\
\hline Sincelejo & 3 & 0,8 \\
\hline Tunja & 5 & 1,3 \\
\hline Yopal & 2 & 0,8 \\
\hline Total & 371 & 100 \\
\hline
\end{tabular}


El 73\% de los dermatólogos que respondieron la encuesta se especializaron en Colombia, los demás se formaron en Brasil, Argentina, Cuba, Guatemala, México, entre otros países. El $27 \%$ de ellos ejerce como especialistas hace 6-10 años y solo el 5\% ejerce hace más de 30 años.

En cuanto a las comorbilidades previas, el 55\% de los encuestados refirió no tener enfermedades subyacentes; sin embargo, 46 tenían antecedentes de asma, lo que representa el $12 \%$ de la población, seguida en frecuencia por la enfermedad tiroidea $(7,3 \%)$ y la hipertensión arterial (HTA) (5,4\%).

Respecto a su lugar de trabajo, de los 371 dermatólogos, la mayoría (79\%) ejerce en su consultorio particular y en hospitales privados (tabla 2). El 67\% trabaja con medicina prepagada o pólizas.

La forma de vinculación laboral más frecuente fue la prestación de servicios, con el 36\% de los encuestados, seguida de los independientes, que representaron el $24,5 \%$ y la combinación de ambas modalidades, con el $17,5 \%$. Menos del 6\% de los encuestados reportó tener un contrato a término indefinido.

Cuando se indagó por el volumen de consulta previo a la pandemia, el 29\% de los encuestados atendían entre 81 y 120 pacientes a la semana y el $25 \%$ entre 51 y 80 pacientes; ambos grupos representaron el 54\% de los dermatólogos. El 69\% realizaba entre 10 y 30 procedimientos dermatológicos/semana y el $42 \%$ realizaba entre 1 y 9 procedimientos dermocosméticos/semana.
Antes de la pandemia, solo el 9\% de los encuestados utilizaba la teledermatología, y la mayoría de ellos lo hacía para la atención de pacientes residentes fuera del país. El tiempo dedicado a esta modalidad de atención, en la mayoría de los casos, fue de 4 a 6 horas semanales.

El 74\% de los encuestados informó que en su lugar de trabajo habían suspendido la consulta de dermatología y solo el 6\% de los dermatólogos continuaron con la atención de pacientes en los servicios de urgencias o interconsultas prioritarias. Estos últimos atendían menos de 20 pacientes a la semana.

Durante el aislamiento preventivo obligatorio, el 83\% de los encuestados reportó la cancelación de los procedimientos dermatológicos electivos, siendo las biopsias de piel el procedimiento más realizado por aquellos que continuaron su ejercicio. Sin embargo, el 96\% reportó hacer menos de tres biopsias a la semana.

El 78\% de los encuestados empezó a utilizar la teledermatología como herramienta laboral después del inicio de la pandemia, y las plataformas preferidas fueron WhatsApp ${ }^{\circledR}$, Zoom ${ }^{\circledR}$ y Skype ${ }^{\circledR}$, con el 47\%, el $26 \%$ y el $11 \%$, respectivamente. El $65 \%$ de los encuestados atendía tanto pacientes nuevos como controles. El 65\% de los dermatólogos que hacen teleconsulta valoran entre 1 y 20 pacientes a la semana, y el tiempo de dedicación a esta actividad es de 20 a 30 minutos, por paciente. El 66\% de los encuestados desarrollan la teleconsulta solo con pacientes particulares, y la principal modalidad de recaudo de honorarios fue la

Tabla 2. Filiación a las diferentes entidades de salud

\begin{tabular}{|c|c|c|}
\hline Variable & Frecuencia & Porcentaje \% \\
\hline Consultorio privado & 293 & 79 \\
\hline Hospital privado & 150 & 40,4 \\
\hline $\begin{array}{l}\text { EPS régimen privado y } \\
\text { contributivo }\end{array}$ & 67 & 18,1 \\
\hline Hospital público & 69 & 18,6 \\
\hline Régimen especial & 14 & 3,8 \\
\hline
\end{tabular}

EPS: entidad promotora de salud. 
transferencia bancaria, seguida de $\mathrm{PayU}^{\circledR}$ y $\mathrm{Nequi}^{\circledR}$; sin embargo, el 2\% reportó no cobrar por los servicios prestados a través de estas plataformas.

El $42 \%$ de los encuestados diligencia una historia clínica electrónica y el proveedor más utilizado para hacer el registro es $\mathrm{HiMed}^{\circledR}$, con el $11 \%$ de los casos. Cuando se preguntó sobre la posibilidad de continuar ejerciendo en la modalidad de teleconsulta, una vez se supere la pandemia, el 39\% respondió afirmativamente, mientras que el $47 \%$ solo la utilizaría en casos especiales. El 57\% aceptaría atender entre 1 y 10 consultas virtuales a la semana.

En cuanto a los elementos de protección personal, el $32 \%$ de los encuestados accedió a todos los EPP necesarios por medio de su empleador, el $27 \%$ los obtuvo solo parcialmente y el 20\% los consiguió por su cuenta.

El 65\% de los encuestados consideró que el país no estaba preparado para enfrentar la pandemia, y cuando se les interrogó sobre la posibilidad de trabajar como voluntarios en la atención de pacientes con COVID-19, el 80\% manifestó que no lo haría y el 91\% refirió encontrarse parcial o totalmente abrumado con la información mediática sobre la pandemia.

También se indagó sobre la presencia de síntomas emocionales relacionados con la pandemia y los más frecuentemente reportados fueron estrés, ansiedad y depresión; sin embargo, el 21\% manifestó no haber presentado ninguno de estos síntomas (tabla 3).

Finalmente, acerca de la disposición para asistir a capacitaciones en línea, el 95\% respondió afirmativamente. El 41\% participaría como capacitador voluntario, el $71 \%$ estaría dispuesto a recibir una visita médica virtual y el $73 \%$ realizaría una teleconsulta gratuita en caso de que sus colegas médicos sufrieran manifestaciones cutáneas asociadas al uso prolongado de EPP.

\section{DISCUSIÓN}

La pandemia por SARS-CoV-2 ha limitado la práctica de las especialidades médicas que demandan contacto cercano con el paciente ${ }^{(1)}$. Durante una consulta dermatológica presencial, el acercamiento físico es inevitable, pues para observar las características de las lesiones y los detalles semiológicos que permiten orientar el diagnóstico clínico se requieren menos de $20 \mathrm{~cm}$ de distancia, y esta medida es mucho menor cuando se utiliza el dermatoscopio ${ }^{(7)}$.

Por lo tanto, los servicios de dermatología de todo el mundo han tenido que transformarse y adaptarse para brindar una atención segura y mitigar el riesgo

Tabla 3. Impacto psicológico de la pandemia entre los dermatólogos

\begin{tabular}{lcc} 
Síntoma & Frecuencia & $\%$ \\
Ninguno & 79 & 21,3 \\
\hline Estrés & 65 & 17,5 \\
Ansiedad & 61 & 16,4 \\
\hline Depresión & 35 & 9,4 \\
Ansiedad y depresión & 40 & 10,7 \\
Estrés, ansiedad y depresión & 32 & 8,6 \\
\hline Estrés y ansiedad & 29 & 7,8 \\
Insomnio & 27 & 7,2 \\
Vértigo & 2 & 0,5 \\
Cefalea & 1 & 0,3 \\
Total & 371 & 100
\end{tabular}




\section{Puntos clave}

- Durante el aislamiento preventivo obligatorio, el 83\% de los dermatólogos encuestados reportó la cancelación de los procedimientos dermatológicos.

- $\quad \mathrm{El} 78 \%$ de los encuestados empezó a utilizar la teledermatología después del inicio de la pandemia, pero solo el $39 \%$ manifestó que continuaría con esta actividad una vez superada la contingencia actual.

- $\quad$ El 91\% de los dermatólogos manifestó sentirse abrumado con la información recibida sobre la pandemia. Se identificaron síntomas de estrés (17\%), ansiedad (16\%), depresión (9\%) y una combinación de estos (27\%).

de contagio ${ }^{(8)}$ por SARS-CoV-2. La implementación de nuevos protocolos de atención, el uso permanente de elementos de protección personal, el distanciamiento físico y la limitación de actividades consideradas no prioritarias no solo han dificultado el ejercicio profesional de los dermatólogos, también ha causado pérdida de sus ingresos ${ }^{(9)}$.

El 74\% de los especialistas encuestados refirió que en su lugar de trabajo habían suspendido la consulta de dermatología durante el confinamiento obligatorio, y el 54\% de ellos pasó de atender entre 51 y 120 pacientes/semana, antes de la pandemia, a no realizar ningún tipo de consulta. Solo el $6 \%$ de ellos continuó atendiendo urgencias dermatológicas e interconsultas intrahospitalarias en armonía con las recomendaciones internacionales ${ }^{(10)}$.

Los costos asociados al cumplimiento de las nuevas normas de bioseguridad generaron gastos imprevistos para el 20\% de los dermatólogos, quienes manifestaron no haber recibido oportunamente los EPP por parte de su empleador o su aseguradora de riesgos profesionales.

La tendencia a la cancelación de los procedimientos electivos, conservando solo aquellos considerados urgentes o prioritarios, se generalizó en los países más afectados por la pandemia ${ }^{(7-11)}$. Lo mismo ocurrió con los procedimientos dermocosméticos, que la literatura recomienda aplazar, particularmente en la población de mayor riesgo, como el láser, los peelings químicos profundos, la fototerapia y los hilos tensores, entre otros, debido al riesgo de transmisión ${ }^{(12)}$ de SARSCoV-2. Esta reducción en la demanda de procedimientos dermatológicos y dermocosméticos fue la que mayor detrimento generó en el ingreso de los dermatólogos colombianos.

El acceso a las plataformas digitales y a los servicios de teleconsulta se incrementó de manera exponencial debido a la necesidad de evitar la interacción personapersona, disminuir el riesgo de contagio y, en forma simultánea, reactivar la atención de enfermedades consideradas no esenciales ${ }^{(10,13)}$. La teleconsulta constituye un acto médico, con todas las consideraciones y consecuencias de cualquier relación médico-paciente, donde el profesional de la salud debe tomar decisiones a partir de la interacción que le proporcionan las diversas plataformas; sin embargo, tanto el profesional como el paciente pueden experimentar las dificultades inherentes a un acto médico a distancia ${ }^{(13)}$.

Teniendo en cuenta que en el momento de responder la encuesta el $78 \%$ de los dermatólogos realizaba teleconsulta, llama la atención que solo el 39\% manifestó que continuaría con esta actividad una vez superada la contingencia actual, probablemente debido a las limitaciones propias de las consultas virtuales ${ }^{(14)}$, tales como: las dificultades técnicas relacionadas con la conectividad, el funcionamiento de las plataformas y el uso de los dispositivos; el detrimento de la relación médico-paciente por percepción de insatisfacción; la menor calidad diagnóstica; el riesgo de pérdida de la privacidad y la confidencialidad; el desconocimiento de los aspectos éticos y médico-legales, entre otras ${ }^{(13}$, 14).

En contraste con lo anterior, el elevado porcentaje (95\%) de dermatólogos interesados en continuar participando en actividades académicas virtuales confirma la tendencia mundial en torno a la implementación 
exitosa de los modelos en línea como nuevo pilar de la educación continuada, tanto para los especialistas, como para los estudiantes de pre y posgrado ${ }^{(8,15)}$.

Finalmente, en esta encuesta se identificó un preocupante riesgo psicosocial, pues además de que el 91\% de los encuestados manifestó sentirse abrumado con la información recibida sobre la pandemia, se identificaron síntomas de estrés (17\%), ansiedad (16\%), depresión ( $9 \%$ ) o una combinación de estos $(27 \%)$, hallazgo previamente descrito entre el personal de la salud durante otros brotes epidémicos ${ }^{(16)}$.

\section{CONCLUSIÓN}

En conclusión, la pandemia por SARS-CoV-2 ha impactado negativamente en la estabilidad laboral y en el ingreso de los dermatólogos colombianos. La situación actual es un gran reto para la especialidad, que no solo debe mitigar el riesgo biológico derivado del retorno a sus actividades presenciales, sino conocer e implementar medidas preventivas que permitan proteger la salud y el bienestar común, mientras se fortalecen las condiciones para un ejercicio seguro y de calidad, apoyado en las nuevas tecnologías.

\section{REFERENCIAS}

1. Gisondi P, Piaserico S, Conti A, Naldi L. Dermatologists and SARS-CoV-2: the impact of the pandemic on daily practice. J Eur Acad Dermatol Venereol. 2020;34(6):1196-201. doi: 10.1111/jdv.16515

2. Ali FR, Al-Niaimi F. Noncutaneous considerations of COVID-19 for dermatology practices. J Cosmet Dermatol. 2020;19(7):1544. doi: 10.1111/jocd.13444

3. Loeb AE, Rao SS, Ficke JR, Morris CD, Riley LH, Levin AS. Departmental experience and lessons learned with accelerated introduction of telemedicine during the COVID-19 crisis. J Am Acad Orthop Surg. 2020;28(11):e469-e76. doi: 10.5435/JAAOS-D-20-00380

4. Bokolo AJ. Exploring the adoption of telemedicine and virtual software for care of outpatients during and after COVID-19 pandemic. Ir J Med Sci. 2020:110. doi: 10.1007/s11845-020-02299-Z

5. Chuchvara N, Patel R, Srivastava R, Reilly C, Rao BK. The growth of teledermatology: Expanding to reach the underserved. J Am Acad Dermatol. 2020;82(4):1025-33. doi: 10.1016/j.jaad.2019.11.055

6. Litchman GH, Rigel DS. The immediate impact of COVID-19 on US dermatology practices.
J Am Acad Dermatol. 2020;83(2):685-6. doi: 10.1016/j.jaad.2020.05.048

7. Wollina U. Challenge of COVID-19 pandemic for dermatology. Dermatol Ther. 2020:e13430. doi: 10.1111/dth.13430

8. Fahmy DH, El-Amawy HS, El-Samongy MA, Fouda AA, Soliman SH, El-Kady A, et al. COVID-19 and dermatology: a comprehensive guide for dermatologists. J Eur Acad Dermatol Venereol. 2020;34(7):1388-94. doi: 10.1111/jdv.16545

9. Campanati A, Brisigotti V, Diotallevi F, D’Agostino G, Paolinelli M, Radi G. Active implicationsfordermatologists in 'SARS-CoV-2 ERA': Personal experience and review of literature. J Eur Acad Dermatol Venereol. 2020;1626-32. doi: 10.1111/jdv.16646

10. Soutou B, Tomb R. The multifaceted of the dermatologist in the Covid-19 pandemic. SN Compr Clin Med. 2020;2:1388-92. doi: 10.1007/s42399-020-00423-9

11. Calvache N, Jaimes JC, Candelo P, Prada C, Pineda Y, Peña E, et al. Perspectiva de la dermatología y COVID-19. Rev Asoc Colomb Dermatol. 2020;28:1726. doi: 10.29176/2590843X.1487

12. Türsen Ü, Türsen B, Lotti T. Aesthetic dermatology procedures in coronavirus-days. J Cosmet Dermatol. 2020;19(8):1822-5. doi: 10.1111/jocd.13509

13. Arimany-Manso J, Pujol RM, García-Patos V, Saigí U, Martin-Fumadó C. Aspectos médico-legales de la teledermatología. Actas Dermosifiliogr. 2020;111(10):815-21. doi: 10.1016/j.ad.2020.08.008

14. Rismiller $\mathrm{K}$, Cartron AM, Trinidad JCL. Inpatient teledermatology during the COVID-19 pandemic. J Dermatolog Treat. 2020;31(5):441-3. doi: 10.1080/09546634.2020.1762843

15. Reinholz M, French L. Medical education and care in dermatology during the SARS-CoV2 pandemia: challenges and chances. J Eur Acad Dermatol Venereol. 2020;34:e214-e215. doi: 10.1111/jdv.16391

16. Preti E, Di Mattei V, Perego G, Ferrari F, Mazzetti M, Taranto P, et al. The psychological impact of epidemic and pandemic outbreaks on healthcare workers: rapid review of the evidence. Curr Psychiatry Rep. 2020;22(8):43. doi: 10.1007/s11920-020-01166-z 